\title{
Los sistemas de salud de Cuba y Uruguay en el contexto de América Latina: una reflexión
}

\author{
Uruguayan and Cuban public health systems \\ in the Latin American context: a consideration
}

M ónica Sans y Pedro C. Hidalgo 1

\footnotetext{
1 Sección Antropología Biológica, Facultad de Humanidades y Ciencias de la Educación, Universidad de la República, Magallanes $1.577,11.200$ msans@adinet.com.uy
}

Abstract At first, we analyze different socioeconomic factors involved in the Latin American health systems, as the application of the neoliberal economic model and the globalization process; these factors make health to be seen as a merchandise. W e discuss to what extent the diverse population groups are affected by the socio-economic factors, and how the latter can harm health as a collective good. Also, we consider the problems caused by the privatization of health, and what happens when the State looses its role as responsible for social security. In a second part, we analize health indicators in Latin America, and make correlation studies to determine associations among them. From the results it is possible to conclude that the health indicators are determined to a great extent by socio-economic factors, and that the health practice should be focused in primary health care and in health education. Finally, we particularly consider the cases of U ruguay and Cuba, two examples of a good performance in health but with great differences in their historical processes and health policies.

Key words U ruguay, Cuba, Latin America, Public health, $\mathrm{H}$ ealth indicators
Resumen Se parte del análisis de diferentes factores soci oeconómicos que han incidido en los sistemas de salud de A mérica Latina, como la aplicación del model o económico neoliberal y el proceso de globalización, que hacen quela salud sea vista como una mercancía. Posteriormente, se discute en qué medida estos factores inciden en diversos sectores de la población, y cómo afectan a la salud como bien colectivo; asimismo, se examinan los problemas que emanan de la privatización de la salud, y qué sucede cuando el Estado pierde su rol de garante de la seguridad social. Se analizan indicadores de salud de América Latina, realizándose estudios de correlación para visualizar sus asociaciones. De estos datos se concluye que los indicadores de salud están determinados en gran medida por los factores socioeconómicos; y que la práctica sanitaria debe centrarse en la atención primaria y dela educación en salud. Por último, se consideran en particular los casos de $U$ ruguay y Cuba, dos ejemplos de buen desempeño en salud aunque con grandes diferencias históricas y de políticas de salud.

Palabras-clave U ruguay, Cuba, América Latina, Salud Pública, Indicadores de salud 


\section{Introducción}

La globalización es un fenómeno complejo que, por sus alcances, es susceptible de diferentes interpretaciones y de abordajes que pueden ser incluso contradictorios, de acuerdo a las premisas de las que se parta y de los intereses que se defiendan. Las implicaciones económicas, financieras, políticas e institucionales son aspectos subyacentes a la globalización que no son, en general, abordados fuera del ámbito de los especialistas y tecnócratas. El análisis de las diversas dimensiones de la globalización está fuera de nuestros propósitos, pero sí es pertinente considerar las visiones economicistas sobre el desarrollo presente y futuro de la salud pública en las que se basan los ideólogos de la globalización.

Las fuerzas de la economía globalizadora de mercado son generadoras de riquezas pero también son generadoras de profundas asimetrías sociales, donde los estratos social es tienden cada vez más a los extremos, de los que pueden (porque tienen) y los que no pueden (porque no tienen).

Los procesos de reforma económica orientados al mercado con el fin de aumentar la competitividad y sanear financieramente el gasto público social han sido parte del paradigma imperante en América Latina en las últimas décadas. La orientación económica neoliberal ha provocado el deterioro de la satisfacción de las necesi dades básicas de los sectores más pobres de la población. El supuesto que el mercado es el único mecanismo idóneo de asignación y distribución de recursos ha llevado a un debilitamiento estructural, con diferencias de grado entre un país y otro, visualizable en la identificación y confección de estrategias de inversión en el sector público, en la regulación del sector privado, y en la formulación y aplicación de una política económica y administrativa en la salud y la enseñanza pública.

El objetivo de este artículo es hacer un análisis en el ámbito de la salud pública de Cuba y de U ruguay dentro del contexto de América Latina.

\section{La salud como mercancía}

Desde la caída del muro de Berlín se ha ido generalizando la visión de que la liberación de los mercados forma un todo con la democracia; en este marco, los objetivos económicos, políticos y sociales de un país quedan subordinados a las leyes de la oferta y la demanda y son independientes de la acción e intervención del estado. De hecho, el mercado nunca ha sido un principio sustentador de la organización social; se ha considerado como signo de modernidad a la posición de defensa extrema del mercado, y concomitantemente, a la retracción del estado, sin pensarse en el precio que el pueblo tiene que pagar (Cardoso, 1995).

La salud es incompatible con un sistema de precios de mercado, ya que no es posible sustentar un sistema de salud con base en el principio de que los servicios que un individuo obtiene sean correlativos a los servicios que el dinero, como bien capital, proporcione. No es posible excluir del acceso a la salud a quienes no tienen medios de subsistencia o no pueden pagar por los servicios asistenciales.

\section{La salud como bien público}

La salud como bien público es un bien colectivo, un derecho de todos que los protagonistas sociales, económicos y políticos de cada país deben defender y preservar. Es un derecho inalienable del pueblo, no negociable y cuyo goce no admite rivalidad.

En una sociedad pluralista y justa, toda persona, independientemente de su posición social, debe tener derecho a la mejor calidad de atención de salud, lo que es posible lograr por la concurrencia de los sectores públicos y privados hacia los intereses de la salud pública como un todo. En la coyuntura económica y política actual, el estado no puede renunciar a su responsabilidad en la organización social. La sustentabilidad social del desarrollo exige la democratización del estado y no su desmantelamiento y sustitución por las fuerzas ciegas del mercado (Guimaraes, 1996).

El modelo económico neoliberal preconiza la reducción de la figura del estado y su capacidad de intervención, lo que favorece la destrucción sistemática de la capacidad de garante de la seguridad social del estado y no permite resolver los problemas planteados de equidad y justicia social. Los grandes intereses económicos y financieros están más interesados en la búsqueda de la llamada eficiencia económica del sector de la salud que en promover su desarrollo en beneficio del pueblo; en pos de esta eficiencia, se transfieren recursos públicos al sector privado, que los asume como costo ad- 
ministrativo con la consecuente mercantilización de la salud.

Al proponerse la privatización de los servicios sociales y de la infraestructura productiva del sector de la salud pública, alentada por los altos costos y la crisis financiera, se entiende que la salud dejará de ser universal y gratuita para pasar a atender los intereses de un sector privilegiado de la sociedad. El sector privado es incapaz de proporcionar por si mismo la cantidad óptima de oportunidades asistenciales; por el contrario, conduce a la distribución asimétrica en los servicios, y, en consecuencia, a un aumento de la pérdida de bienestar en los sectores más pobres. El confiar en la capacidad del sector privado para proporcionar un sistema de salud estable y rentable en situaciones de inestabilidad económica es una fal acia. La práctica demuestra que la regla ha sido brindar un servicio mínimo con un margen de beneficio máximo, produciendo profundas disparidades en la calidad de vida de los distintos estratos de la población, con especial impacto en niños y mujeres que viven en situación de pobreza crítica, y con aumento de la marginalización y el deterioro social.

\section{La salud en el contexto de América Latina}

Cuando se mide cuantitativamente el desarrollo económico y social de los países latinoamericanos por sus respectivos indicadores económicos y de salud, se obtiene una visión de conjunto que permite apreciar los niveles al canzadosy las tendencias predominantes (ver Tabla 1).

Un análisis de los coeficientes de correlación entre algunos de estos indicadores en la valorización del estado sanitario de los países latinoamericanos permite ver la relación entre los factores económicos y sociales. Por ejemplo, se puede observar que la mortalidad infantil está fuertemente asociada con el nivel de alfabetización ( $r=0.790$ ), el acceso al agua potable ( $r=0.763)$ y la urbanización $(r=0.603)$, más que con el número de médicos por habitante $(r=0.396)$ o el de camas hospitalarias ( $r=$ $0.517)$. Con base en estos resultados puede establecerse que los indicadores de salud están determinados en gran medida por los factores socioeconómicos; esto apoya la conclusión de del Caño (2000) acerca de que el enfoque de la práctica sanitaria debe estar en la atención primaria y de la educación en salud, elementos tomados por la medicina preventiva.
Nadie puede desconocer el mejoramiento experimentado por América Latina en los últimos años, que se traduce por ejemplo en un notorio descenso de la tasa de mortalidad infantil: de acuerdo a datos de la OPS (1998), la disminución porcentual de la tasa entre 19501955 y 1990-1995 fue superior al 80\% en Chile, Costa Rica y Cuba, entre $60 \%$ y $79 \%$ en Argentina, Brasil, Colombia, Ecuador, El Salvador, Guatemala, Haití, H onduras, M éxico, Nicaragua, Panamá, Perú, República Dominicana, U ruguay y $V$ enezuela, y entre 40 y $49 \%$ en Bolivia y Paraguay. Sin embargo, el $75 \%$ de los países tiene aún una tasa de mortalidad infantil superior a la de 20 por cada 1.000 nacidos vivos.

También, el 75\% de los países latinoamericanos tiene tasas de mortalidad materna superiores a 40 por cien mil. El análisis de coeficientes de correlación de éstas con los indicadores mostrados en la tabla 1 muestra que hay una alta correlación con el índice de pobreza ( $r=$ 0.701 ) y también con la tasa de mortalidad infantil $(r=0.858)$.

Es de señalar que en algunos países el porcentaje del producto bruto interno (PBI) dedicado a la salud no guarda relación con los resultados de sus indicadores de salud, como es el caso de la Argentina y El Salvador; estos países son un ejemplo de aquéllos que gastan mucho y mal en la salud pública, con la diferencia de que la Argentina tiene una menor pobreza y un mejor nivel per capita.

\section{Situación actual de salud en Cuba y Uruguay}

La situación de la salud en Cuba y U ruguay deriva de procesos diferentes y antagónicos. El U ruguay, a comienzos de siglo y hasta aproximadamente 1950, tenía la tasa de mortalidad infantil más baja de Latinoamérica (concretamente, entre 1950 y 1955 al canzaba el 57 por mil, seguida por Argentina con 66 por mil) (OPS, 1998). Posteriormente, si bien la tasa de mortalidad infantil continuó bajando, otros países de la región obtuvieron mejores resultados; a fines de la década de 1980, luego de 12 años de dictadura cívico-militar, U ruguay había pasado al sexto lugar con relación a este indicador. Por el contrario, Cuba, que tenía hacia 1950 una tasa de mortalidad infantil de 81 por mil, hacía 1990 logrará reducirla a 10 por mil (OPS, 1998) 
Tabla 1

Indicadores sociales, económicos y de salud de América Latina.

\begin{tabular}{|c|c|c|c|c|c|c|c|}
\hline País & $\begin{array}{l}\text { Población } \\
\text { x } 10^{6}\end{array}$ & $\begin{array}{l}\text { Nivel de } \\
\text { Urbaniza- } \\
\text { cion }\end{array}$ & $\begin{array}{l}\text { PBI per } \\
\text { cápita } \\
\text { (dólares) }\end{array}$ & $\begin{array}{l}\% \text { PBI } \\
\text { en Salud }\end{array}$ & $\begin{array}{l}\% \text { agua } \\
\text { potable }\end{array}$ & $\begin{array}{l}\text { \% de alfa- } \\
\text { betización }\end{array}$ & $\begin{array}{l}\text { Esperanza } \\
\text { de vida } \\
\text { femenina }\end{array}$ \\
\hline Argentina & 37.0 & 86 & \multirow[t]{21}{*}{8380} & 8.1 & 65 & 96 & 78 \\
\hline Bolivia & 8.3 & 51 & & 5.0 & 61 & 83 & 64 \\
\hline Brasil & 170.4 & 75 & & 6.9 & 69 & 85 & 72 \\
\hline Chile & 15.2 & 85 & & 7.5 & 85 & 95 & 80 \\
\hline Colombia & 42.1 & 70 & & 9.3 & 80 & 87 & 75 \\
\hline Costa Rica & 4.0 & 47 & & 6.8 & 100 & 95 & 79 \\
\hline Cuba & 11.1 & 74 & & 6.4 & 92 & 97 & 78 \\
\hline República & 8.3 & 60 & & 6.5 & 65 & 82 & 72 \\
\hline \multicolumn{7}{|l|}{ Dominicana } & \\
\hline Ecuador & 12.6 & 56 & & 3.6 & 70 & 90 & 74 \\
\hline El Salvador & 6.2 & & & & & & \\
\hline \multicolumn{7}{|l|}{ Guatemala } & \\
\hline \multicolumn{7}{|l|}{ H aití } & \\
\hline \multicolumn{7}{|l|}{ Honduras } & \\
\hline \multicolumn{7}{|l|}{ M éxico } & \\
\hline \multicolumn{7}{|l|}{ Nicaragua } & \\
\hline \multicolumn{7}{|l|}{ Panamá } & \\
\hline \multicolumn{7}{|l|}{ Paraguay } & \\
\hline \multicolumn{7}{|l|}{ Perú } & \\
\hline \multicolumn{7}{|l|}{ Uruguay } & \\
\hline Venezuela & & & & & & & \\
\hline
\end{tabular}

\begin{tabular}{|c|c|c|c|c|c|c|c|}
\hline País & $\begin{array}{l}\text { Esperanza } \\
\text { de vida } \\
\text { masculina }\end{array}$ & $\begin{array}{l}\text { Tasa de } \\
\text { mortalidad } \\
\text { infantil }\end{array}$ & $\begin{array}{l}\text { Tasa de } \\
\text { mortalidad } \\
\text { menores } \\
\text { de } 5 \text { años }\end{array}$ & $\begin{array}{l}\text { Tasa de } \\
\text { mortalidad } \\
\text { materna }\end{array}$ & $\begin{array}{l}\text { Médicos } \\
\text { por } 1.000 \\
\text { habitantes }\end{array}$ & $\begin{array}{l}\text { Camas en } \\
\text { hospitales } \\
\text { por } 1.000 \\
\text { habitantes }\end{array}$ & $\begin{array}{l}\% \text { población } \\
\text { en pobreza }\end{array}$ \\
\hline $\begin{array}{l}\text { Argentina } \\
\text { Bolivia } \\
\text { Brasil } \\
\text { Chile } \\
\text { Colombia } \\
\text { Costa Rica } \\
\text { Cuba } \\
\text { República } \\
\text { Dominicana } \\
\text { Ecuador } \\
\text { El Salvador } \\
\text { Guatemala } \\
\text { Haití } \\
\text { Honduras } \\
\text { M éxico } \\
\text { Nicaragua } \\
\text { Panamá } \\
\text { Paraguay } \\
\text { Perú } \\
\text { Uruguay } \\
\text { Venezuela }\end{array}$ & $\begin{array}{l}70 \\
61 \\
65 \\
73 \\
67 \\
73 \\
74 \\
66 \\
68\end{array}$ & 20,9 & 24,3 & 44 & 24,9 & 44,8 & 25 \\
\hline
\end{tabular}


Los resultados alcanzados en la actualidad en el sector de la salud por estos dos países latinoamericanos (ver Tabla I), a pesar de sus diferencias en cuanto a los procesos históricos, forma de gobierno y organización social, muestran que la satisfacción de necesidades básicas por parte de la atención de la salud pública rinde indicadores positivos, como puede verse en una mortalidad infantil más baja y una longevidad mayor que en la mayoría de países latinoamericanos; entre estas necesidades básicas pueden mencionarse el abastecimiento adecuado de agua potable, el saneamiento básico, la asistencia materno infantil, la inmunización contra las principales enfermedades infecciosas y los programas de atención de las enfermedades comunes.

Sin embargo, cuando el análisis de la situación de la salud pública penetra más allá de los indicadores aparecen otras realidades. En el U ruguay, de acuerdo a datos del Instituto $\mathrm{Na}$ cional de Estadística (1996) en cuanto a la cobertura de salud de la población, puede observarse que el $40 \%$ depende del sector público, el $48 \%$ pertenece al sector mutual y privado, y aproximadamente el $12 \%$ carece de cobertura de salud. En las áreas rurales la asistencia pública cubre el $42 \%$ de la población, la privada el $39 \%$ y el $19 \%$ no tiene cobertura de salud; de este último grupo, el 31\% son menores de 15 años. Busto (1992) señala que la cifra global de la tasa de mortalidad infantil en el año 1992 era de 21 por mil nacidos vivos, pero era de 28 por mil en el sector de atención pública y solo de 10 por mil en el sector mutual y privado, lo que refleja la situación de deterioro y empobrecimiento en que vive un sector importante de la población uruguaya.

Un nuevo elemento a considerar en el Uruguay es que los servicios de salud pública afrontan actualmente un aumento en su demanda, derivado de la crisis económica y el empobrecimiento de la población, lo que conduce a un deterioro de la cobertura asistencial que afecta las clases sociales más necesitadas. Aún cuando el Ministerio de Salud Pública ha implementado programas nacionales de salud asumiendo la coordinación entre el sector público y privado, los mismos adolecen de una efectiva integración de efectores y de redes interactuantes. En la práctica el sistema de salud se muestra fragmentado y desarticulado, sin una efectiva integración sectorial, con una priorización de la atención institucional de alta tecnología, y sin lograr desarrollar los servicios de salud de primer nivel de atención.
De acuerdo a datos de la OPS (1999), U ruguay es el país de Latinoamérica que gasta mayor porcentaje del PBI en salud, pero este gasto no se refleja claramente en los estratos que recurren al sector público. Debemos coincidir con Portillo (1999) cuando expresa que la mayor eficacia en el arte médico se logra con medidas simples y poco costosas; los países que han superado a U ruguay en la mejora de la tasa de mortalidad infantil tienen sistemas de salud nacionales con un fuerte desarrollo de los servicios asistenciales de primer nivel y donde el estado ha asumido claramente el papel de regulador.

En Cuba, las mejoras en el cuidado de la salud pública en las últimas tres décadas han sido parte de un proyecto social que implicó una transformación política y económica de toda la estructura del país. El estado ha asumido totalmente la responsabilidad de la atención de salud y en correspondencia con esto, se asegura su gratuidad y accesi bilidad completa a la totalidad de sus ciudadanos, independientemente de su condición social y nivel de ingreso. Como parte de la estrategia de desarrollo de la salud pública cubana se han delineado los principales esfuerzos en los programas preventivos que abarcan todas las etapas de la vida y en el fortalecimiento de la atención primaria. Uno de los logros más importante al canzados por la salud pública ha sido la integración de los componentes asistenciales, la formación del personal médico y paramédico, y la investigación médica dentro del Sistema Nacional de Salud, que es dirigido por el M inisterio de Salud Pública.

Cuba, a pesar de su difícil coyuntura económica actual, ha podido mejorar sus indicadores de salud. Cabe preguntarse hasta qué punto el deterioro de la alimentación, la escasez de recursos de primera necesidad, y la contracción de su economía permitirán mantener esas tasas, y también por qué no se ha logrado bajar la mortalidad materna al mismo nivel de los otros indicadores de salud. Además, el modelo de sistema de salud cubano está en la coyuntura de cómo logrará salvar la contradicción en la implementación práctica de una medicina cada vez más comunitaria con la medicina de alta tecnología, sin perder su estrategia sanitaria. 


\section{Referencias bibliográficas}

Busto R 1999. Presentación. Atención primaria de salud p.1. Documento № 16. M inisterio de Salud Pública. República Oriental del U ruguay. M ontevideo.

Cardoso FH 1995. Estado, mercado, democracia. ¿Existe una perspectiva latinoamericana? Socialismo y Participación 71:85-94.

Del Caño E 2000. M edicina preventiva. M edicina asistencial. Investigación clínica, pp. 77-88. En Fundamentación y contenido de la medicina antropológica. Sociedad Argentina de Antropología M édica y M edicina Antropológica (ed.). Publicación I. Buenos Aires.

Guimaraes RP 1996. Globalización, actores sociales y democracia en América Latina. Revista de Humanidades y Ciencias Sociales, Segunda Epoca 2(2):31-52.

Instituto Nacional de Estadística 1996. VII Censo General de Población, III de Hogares y V de Viviendas, 1996. República Oriental del U ruguay. M ontevideo.

OPS 1998. La salud en las Américas. Publicación Científica No 569, Vol.1, 380 pp. Oficina Sanitaria Panamericana. Washington DC.

OPS 1999. Perfiles básicos de salud de países en las Américas. Resumen, 1999. http://www. paho.org/Spanish/ sha/perfiles.htm.

Portillo J 1999. Arte médico y responsabilidad humana. Salud Problema. Nueva Epoca Año 4(6):7-16. 